\title{
Локально деформированные структуры Ge/SOI с улучшенным теплоотводом как активная среда для кремниевой оптоэлектроники
}

\author{
(C) Д.В. Юрасов ${ }^{1}$, Н.А. Байдакова ${ }^{1}$, В.А. Вербус ${ }^{1,2}$, Н.С. Гусев ${ }^{1}$, А.И. Машин ${ }^{3}$, Е.Е. Морозова ${ }^{1}$, \\ А.В. Нежданов ${ }^{3}$, А.В. Новиков ${ }^{1,3}$, Е.В. Скороходов ${ }^{1}$, Д.В. Шенгуров ${ }^{1}$, А.Н. Яблонский ${ }^{1}$ \\ ${ }^{1}$ Институт фозики микроструктур Российской академии наук, \\ 603087 д. Афонино, Нижегородская обл., Россия \\ ${ }^{2}$ НИУ Высшая школа экономики, \\ 603155 Нижний Новгород, Россия \\ ${ }^{3}$ Нижегородский государственный университит им. Н.И. Лобачевского, \\ 603950 Нижний Новгород, Россия \\ E-mail: Inquisitor@ipmras.ru
}

Поступила в Редакцию 24 апреля 2019 г.

В окончательной редакции 29 апреля 2019 г.

Принята к публикации 29 апреля 2019 г.

Представлены результаты по формированию локально растянутых Ge микроструктур на подложках SOI (silicon-on-insulator) и исследованию их оптических свойств. Свободновисящие Ge структуры были получены с помощью оптической литографии, плазмохимического и селективного химического травления с использованием метода „концентрации напряжений“. Для обеспечения теплоотвода от Ge микроструктур схема их формирования была модифицирована таким образом, чтобы обеспечить механический контакт подвешенной части микроструктуры с нижележащими слоями. Для реализации вышеуказанной схемы были использованы подложки SOI с тонким верхним слоем $\mathrm{Si}$, толщина которого составляла 100 нм. Уменьшение локального разогрева в подобных структурах было показано с помощью измерений спектров комбинационного рассеяния света в зависимости от мощности накачки. Измерения спектров микрофотолюминесценции показали значительное возрастание интенсивности сигнала в растянутых областях Ge микроструктур, а также возможность увеличения максимальной мощности оптической накачки (не приводящей к необратимым изменениям) для микроструктур, в которых обеспечен механический контакт растянутой части с нижележащими слоями, по сравнению со свободновисящими структурами.

Ключевые слова: Si структуры, молекулярно-пучковая эпитаксия, деформация, адгезия, теплоотвод, фотолюминесценция.

DOI: $10.21883 /$ FTP.2019.10.48290.36

\section{1. Введение}

Как известно, одной из основных задач, решение которых необходимо для дальнейшего развития кремниевой оптоэлектроники, является формирование на кремнии коммерчески доступного эффективного источника излучения ближнего инфракрасного (ИК) диапазона. Одним из вариантов решения данной задачи является использование деформированного Ge в качестве активной среды. Объемный $\mathrm{Ge}$ является непрямозонным полупроводником и поэтому эффективность излучательной рекомбинации носителей заряда в нем невысока. Однако разница между значениями ширины прямой и непрямой запрещенных зон в $\mathrm{Ge}$ составляет всего 140 мэВ при $300 \mathrm{~K}$, и эта разница может быть уменьшена за счет деформации растяжения. Как показывают оценки, приведенные в литературе, при приложении двуосного растяжения $1.5-1.8 \%$ или одноосного растяжения вдоль направления [100] в 4.5-5\% данная разница обращается в нуль [1-4]. Столь высокие значения напряжений могут быть достигнуты при эпитаксиальном росте Ge на слоях с большим параметром решетки, таких как InGaAs или $\mathrm{GeSn}[5,6]$. Однако такой подход, с одной стороны, тре- бует выращивания толстых релаксированных буферных слоев, что усложняет процесс изготовления и удорожает всю технологию, а с другой - позволяет формировать только тонкие бездефектные пленки растянутого $\mathrm{Ge}$, толщиной меньше критической толщины псевдоморфного роста (единицы нм).

Другим возможным методом получения высоких деформаций растяжения является использование подхода, в котором искомые значения растяжения достигаются не во всей пленке $\mathrm{Ge}$, a в некоторых локальных областях. Одним из методов формирования локально деформированных $\mathrm{Ge}$ структур является так называемый метод концентрации напряжений, изначально предложенный в [7] для формирования деформированных $\mathrm{Si}$ нанопроволок и позднее реализованный той же группой для гетеросистемы $\mathrm{Ge} / \mathrm{Si}$ [8]. Суть метода заключается в перераспределении упругих деформаций в некоторой области исходной слабо деформированной пленки и создании малых (по сравнению с размерами исходной области) участков с многократно увеличенными значениями деформации [8]. Несмотря на такие преимущества данного подхода, как относительная простота реализации, возможность его применения для различных 
материальных систем, легкость контроля за величиной получающихся деформаций и др., ему присуща проблема с отводом тепла от сформированных микроструктур в силу самой его идеи (в методе формируются так называемые свободновисящие структуры, теплоотвод от которых априори мал). Отчасти с проблемой теплоотвода связывалось долгое отсутствие наблюдения стимулированного излучения в подобных структурах, несмотря на достижение теоретически предсказанных значений упругих деформаций, при которых оно должно реализовываться [9-12]. Лишь в недавних работах было доложено о достижении лазерной генерации в локально растянутых Ge структурах в геометрии типа „микромостик“ [13] и „микродиск“ [14] при криогенных температурах, одним из условий достижения которой как раз и была реализация теплоотвода от активной области структур. В обоих случаях для этой цели использовался метод „сращивания“ („,bonding“). Однако для локально растянутых $\mathrm{Ge}$ микроструктур может быть использован другой подход для улучшения теплоотвода, основанный на формировании за счет адгезии механического контакта локально деформированной области структуры с подложкой [15]. В этом случае движущей силой для адгезии выступают капиллярные силы [16]. В настоящей работе представлены результаты использования подобного подхода к улучшению теплоотвода от локально деформированных $\mathrm{Ge}$ микроструктур, а также результаты исследований люминесцентных свойств сформированных структур.

\section{2. Методика эксперимента}

Рост исходных слоев Ge был выполнен методом молекулярно-пучковой эпитаксии. Для реализации адгезии локально деформированных структур в качестве подложек использовались структуры кремний-на-окисле (silicon-on-insulator, SOI) с малой толщиной слоя $\mathrm{Si}(100 \mathrm{Hм})$ над захороненным окислом (рис. 1, $a)$. Для снижения плотности дефектов в слоях Ge/SOI использовался так называемый двухтемпературный метод роста с последующим постростовым циклическим отжигом выращенных структур в вакуумной камере $[17,18]$. За счет оптимизации параметров роста и отжига в полученных Ge буферах была достигнута плотность прорастающих дислокаций на уровне $(2-5) \cdot 10^{7} \mathrm{~cm}^{-2}$ при толщине буфера $\sim 500$ нм [19]. На данных буферах выращивались слои $\mathrm{Ge}$ толщиной 350-400 нм, легированные $\mathrm{Sb}$ на уровне $(1-3) \cdot 10^{19} \mathrm{~cm}^{-3}$ с использованием ранее развитого авторами метода легирования Ge сегрегирующими примесями [20]. Применение легирования Ge сурьмой обусловлено результатами многочисленных исследований, проведенных в том числе и авторами настоящей работы, которые показали, что легирование $\mathrm{Ge}$ донорами способствует увеличению эффективности излучательной рекомбинации носителей заряда в нем [21-26]. По данным рентгенодифракционного анализа, исходные сплошные пленки $\mathrm{Ge}$ обладали двуосной деформацией растяжения на уровне $0.20-0.25 \%$, связанной с разницей коэффициентов температурного расширения $\mathrm{Si}$ и $\mathrm{Ge}$.

Создание локально деформированных Ge микроструктур в геометрии типа „микромостик“ осуществлялось методами оптической литографии, плазмохимического и селективного химического травления. На первом этапе с помощью безмасочной лазерной литографии на установке Heidelberg uPG 101 в фоторезисте AZ1512 формировался необходимый рисунок микромостиков. На последующем этапе в смеси газов $\mathrm{SF}_{6} / \mathrm{C}_{4} \mathrm{~F}_{8}$ с использованием фоторезиста в качестве маски выполнялось плазмохимическое травление структуры, условия которого были подобраны таким образом, чтобы обеспечить высокую анизотропию травления в вертикальном направлении [27]. Таким образом, в результате формировалась нужная геометрия микроструктуры и осуществлялся доступ к слою $\mathrm{Si}$ под слоем $\mathrm{Ge}$. Это позволяло формировать свободновисящую локально деформированную $\mathrm{Ge}$ микроструктуру за счет селективного травления слоя $\mathrm{Si}$, расположенного между слоем Ge и слоем захороненного окисла, в 5\%-м растворе ТМАН. Как было указано выше, для формирования структур, в которых локально растянутая область приводилась в контакт с нижележащим слоем $\mathrm{SiO}_{2}$, использовались подложки SOI с тонким верхним слоем $\mathrm{Si}$ (рис. 1,a). Кроме этого была модернизирована сама схема формирования локально растянутых структур за счет добавления после этапа селективного травления $\mathrm{Si}$ этапа, включающего в себя кратковременное $(\sim 30 \mathrm{c})$ погружение структуры в изопропиловый спирт и последующую ее сушку при температуре $T \approx 50^{\circ} \mathrm{C}$. При этом механический контакт между мостиком и нижележащим слоем $\mathrm{SiO}_{2}$ возникал за счет адгезии при испарении слоя жидкости между ними (рис. $1, b)$.

Полученные $\mathrm{Ge}$ микроструктуры анализировались с помощью сканирующей электронной микроскопии (СЭМ, микроскоп Carl Zeiss Supra 50VP), спектроскопии комбинационного рассеяния света (микро-КРС, зондовая нанолаборатория NTEGRA Spectra), а также спектроскопии микрофотолюминесценции при комнатной температуре (микро-ФЛ). При исследовании микро-ФЛ структуры возбуждались непрерывным излучением второй гармоники $\mathrm{Nd}$ : YAG-лазера (532 нм), которое фокусировалось на образец с помощью объектива $\times 50$ (диаметр пятна $\sim 2$ мкм). Этот же объектив использовался для сбора излучения исследуемых микроструктур. Регистрация спектров микро-ФЛ осуществлялась с помощью решеточного монохроматора Acton 2300i и многоканального детектора OMA-V на основе линейки фотодиодов InGaAs (рабочий диапазон длин волн 0.8-2.2 мкм).

\section{3. Результаты и их обсуждение}

B настоящей работе были сформированы Ge микромостики, причем часть из них была изготовлена сво- 

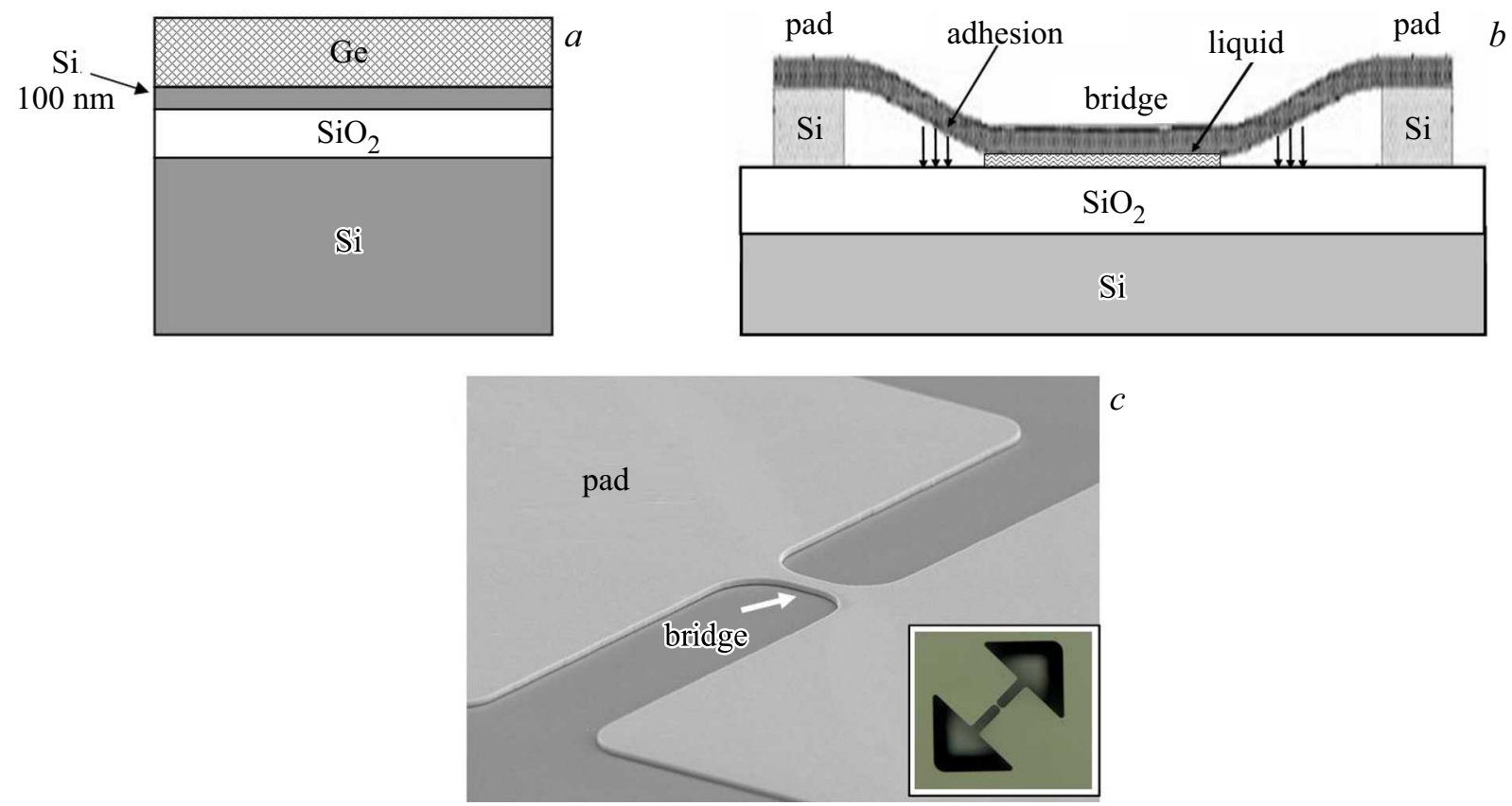

Рис. 1. $a-$ исходная структура для формирования микромостиков, $b-$ схематическое изображение модификации метода формирования микромостика для реализации его адгезии к нижележащему слою, $c-$ СЭМ-снимок мостика с адгезией к нижнему слою. На вставке $(c)$ представлено для наглядности изображение всей структуры с микромостиком.

бодновисящими, а часть - по описанной выше модифицированной схеме (рис. $1, b$ ), в которой мостики приводились в контакт с нижележащими слоями за счет адгезии. Подробное описание геометрических параметров свободновисящих мостиков и анализ распределения упругих напряжений в них были приведены в предыдущей работе авторов [27]. На рис. 1, с показан также СЭМ-снимок мостиков такого типа.

Условия измерения и геометрические параметры мостиков оказались таковыми, что однозначного вывода о наличии/отсутствии контакта мостика с нижележащим слоем $\mathrm{SiO}_{2}$ из данных СЭМ сделать невозможно (рис. 1,c). В связи с этим информация о контакте мостика с нижними слоями и об эффективности теплоотвода от него была получена с помощью измерений микро-КРС и микро-ФЛ. Известно, что деформация $\varepsilon$ в одноосно деформированных вдоль направления [100] Ge микроструктурах может быть вычислена по смещению $\Delta \omega$ пика LO-фонона Ge относительно его положения в объемном, недеформированном $\mathrm{Ge}\left(\sim 300 \mathrm{~cm}^{-1}\right)$ по формуле $\varepsilon=0.68 \Delta \omega-0.019 \Delta \omega^{2}$, учитывающей некоторое отклонение от линейной связи $\varepsilon$ с $\Delta \omega$ [28]. Однако изменение температуры также влияет на положение линий в спектрах комбинационного рассеяния полупроводников $[29,30]$. В связи с этим для оценки величины деформации в микромостиках использовались достаточно малые мощности зондирующего лазерного пучка, при которых локальный разогрев отсутствует (рис. 2,a). Были получены значения одноосной деформации на уровне $\varepsilon=2.15 \%$, что на порядок превышает исходную (но двуосную) деформацию в сплошной пленке и хорошо согласуется с результатами численных расчетов распределения деформации. Измерение же зависимости положения пика LO-фонона от мощности зондирующего лазерного пучка позволило качественно сравнить эффективности теплоотвода от свободновисящих мостиков и от мостиков, сформированных по модернизированной схеме с адгезией к нижнему слою $\mathrm{SiO}_{2}$ (рис. 2, b).

Легко видеть, что с ростом мощности зондирующего лазера появляется дополнительное смещение положения пика LO-фонона в области микромостика, однако для структур с адгезией к нижнему слою оно заметно меньше (рис. $2, b$ ) и сравнимо со смещением пика LO-фонона от исходной пленки Ge. Так, на основе данных по смещению линии от температуры, приведенных в [30], можно оценить величину нагрева микромостика (для максимальной использованной мощности зондирующего лазера $\sim 5.8 \mathrm{мBT)} \mathrm{как} \sim 110 \mathrm{~K}$ для свободновисящих мостиков и $\sim 20 \mathrm{~K}$ для мостиков в контакте с нижним слоем. Данный факт свидетельствует о том, что модернизированная схема формирования локально растянутых $\mathrm{Ge}$ микроструктур действительно позволяет привести активную область структуры в контакт с нижним слоем и улучшить тем самым теплоотвод от нее. Отметим также, что адгезия мостиков к нижележащему слою практически не сказывается на величине деформации в нем, что связывается с незначительным изменением геометрических размеров мостика при этом процессе по сравнению с размерами самого мостика и прилегающих областей.

Улучшение теплоотвода в Ge микромостиках, контактирующих с подложкой, было также подтверждено с помощью измерений спектров микро-ФЛ при различной 

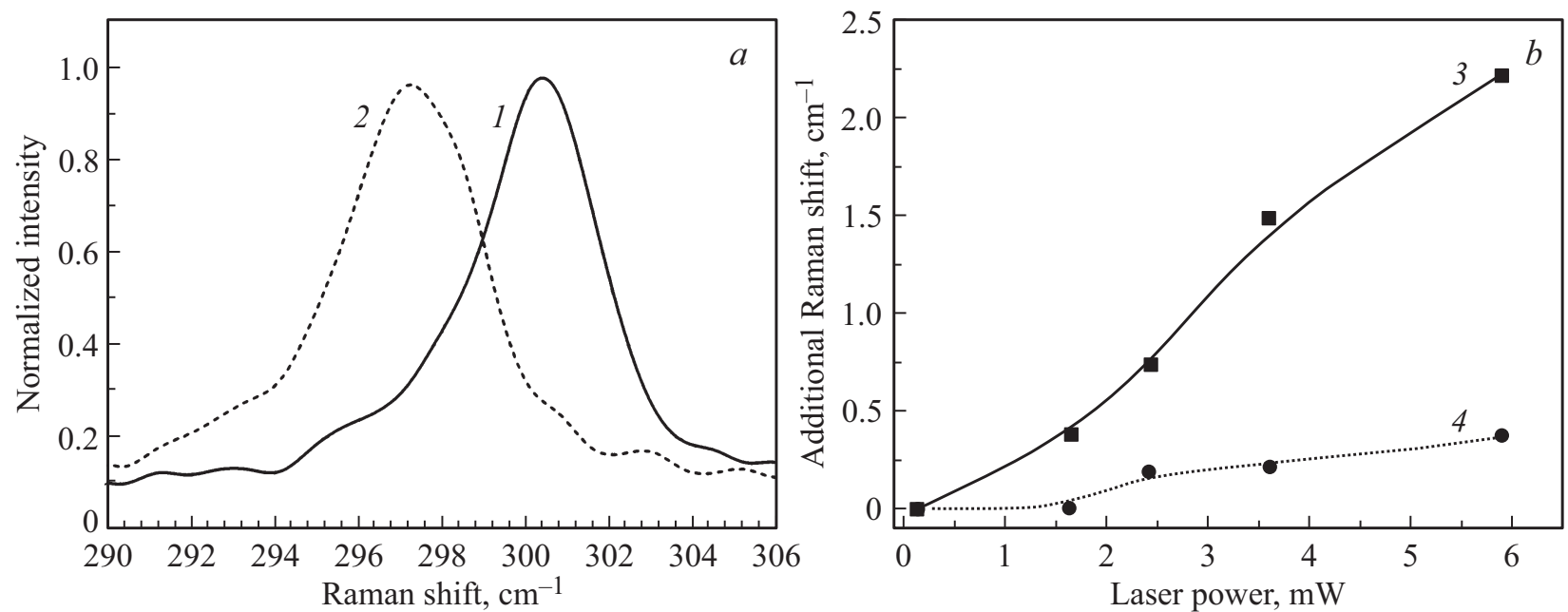

Pис. 2. $a$ - линии LO-фонона в спектре микро-КРC, снятые в центре микромостика $(1)$ и пик от объемного Ge $(2)$. $b-$ дополнительный сдвиг линии LO-фонона, соответствующего 2 (см. $a$ ) при увеличении мощности зондирующего лазера для свободновисящего мостика (3) и для мостика в контакте с нижним слоем $\mathrm{SiO}_{2}(4)$.
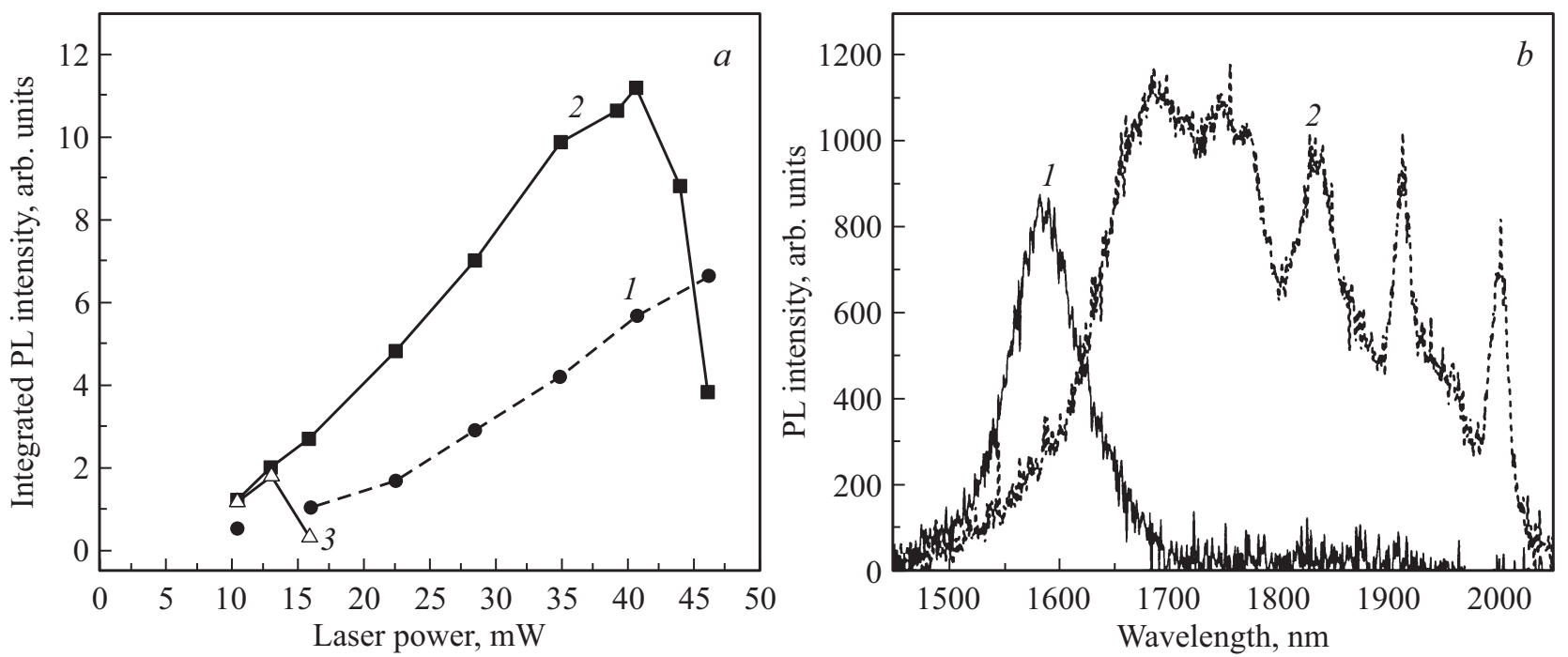

Рис. 3. $a-$ зависимости интегральной интенсивности фотолюминесценции (PL) от мощности оптической накачки для исходной пленки $\mathrm{Ge}(1)$, микромостика в контакте с нижележащим слоем $\mathrm{SiO}_{2}(2)$ и свободновисящего микромостика (3). $b-$ спектры микро-ФЛ от исходной пленки $\mathrm{Ge}(1)$ и от микромостика в контакте с нижележащим слоем $\mathrm{SiO}_{2}(2)$.

мощности оптической непрерывной накачки. Выявлено, что максимальная плотность мощности накачки, при которой еще не происходит разрушения подобных микромостиков из-за их нагрева, составляет $300-500 \mathrm{\kappa BT} / \mathrm{cm}^{2}$, что в 4-5 раз выше, чем для свободновисящих микромостиков (рис. 3, $a$, кривые 2 и 3). Это свидетельствует о том, что даже контакт мостика со слоем $\mathrm{SiO}_{2}$, теплопроводность которого относительно невелика, уже значительно улучшает теплоотвод от него. Возможным шагом по дальнейшему улучшению теплоотвода может служить модернизация схемы формирования мостиков, в которой „подвешивание“ структуры будет осуществляться путем удаления тонкого слоя захороненного окисла в подложках SOI, за счет чего адгезия мостика будет реализована к самой подложке $\mathrm{Si}$, теплопроводность которой более чем на порядок выше, чем $\mathrm{SiO}_{2}$.

Исследование люминесценции сформированных локально растянутых $\mathrm{Ge}$ микроструктур методом спектроскопии микро-ФЛ при комнатной температуре показало возрастание интегральной интенсивности сигнала ФЛ в области мостиков, а также его сдвиг в область бо́льших длин волн (рис. $3, b)$. Полученные данные находятся в качественном соответствии с теоретическими представлениями об изменении зонной структуры Ge при его растяжении. B Ge микромостиках с адгезией к нижележащему слою $\mathrm{SiO}_{2}$ интегральная интенсивность сигнала ФЛ возрастает в $\sim 4.5$ раза по сравнению с сигналом от исходной пленки $\mathrm{Ge}$ (рис. $3, b$ ). Отметим также, что 
в спектре излучения от мостика (рис. $3, b$, кривая 2) можно наблюдать несколько пиков, появление которых связывается с интерференционными эффектами. Однако детальное происхождение этих интерференционных пиков лежит за рамками настоящей работы.

Таким образом, результаты исследований оптических свойств локально растянутых Ge структур в совокупности с возможностью реализации эффективного отвода тепла от них за счет реализации их контакта с подложкой свидетельствуют о перспективности таких структур для создания на их основе $\mathrm{Si}$-совместимых излучателей ближнего ИК диапазона.

\section{4. Заключение}

В работе представлены результаты по формированию локально растянутых $\mathrm{Ge}$ микроструктур на подложках SOI и исследованию их оптических свойств. Показано, что использование подложек SOI с тонким слоем $\mathrm{Si}$ над слоем захороненного $\mathrm{SiO}_{2}$ для роста исходных пленок $\mathrm{Ge}$ и относительная простая модификация схемы формирования локально растянутых микромостиков позволяет обеспечить механический контакт подвешенной части микроструктур с нижележащими слоями. Последнее обеспечивает значительное улучшение теплоотвода от них без заметных изменений в распределении упругих деформаций. Измерения спектров микрофотолюминесценции показали значительное возрастание интенсивности сигнала в растянутых областях $\mathrm{Ge}$ микроструктур по сравнению с исходной пленкой $\mathrm{Ge}$, а также возможность увеличения максимальной мощности оптической накачки (не приводящей к необратимым изменениям) для микроструктур, в которых обеспечен механический контакт растянутой части с нижележащими слоями, по сравнению со свободновисящими структурами.

\section{Финансирование работы}

Работа выполнена при финансовой поддержке Российского научного фонда (грант № 17-72-10207) с использованием оборудования Центра коллективного пользования „Физика и технология микро- и наноструктур“ ИФМ РАН.

\section{Конфликт интересов}

Авторы заявляют, что у них нет конфликта интересов.

\section{Список литературы}

[1] M. El Kurdi, G. Fishman, S. Sauvage, P. Boucaud. J. Appl. Phys., 107, 013710 (2010).

[2] M. Virgilio, C.L. Manganelli, G. Grosso, G. Pizzi, G. Capellini. Phys. Rev. B, 87, 235313 (2013).

[3] C. Boztug, J.R. Sanchez-Perez, F. Cavallo, M.G. Lagally, R. Paiella. ACS Nano, 8, 3136 (2014).

[4] R. Geiger, T. Zabel, H. Sigg. Front. Mater., 2: 52 (2015).
[5] Y. Huo, H. Lin, R. Chen, M. Makarova, Y. Rong, M. Li, T.I. Kamins, J. Vuckovic, J.S. Harris. Appl. Phys. Lett., 98, 011111 (2011)

[6] J. Menéndez, J. Kouvetakis. Appl. Phys. Lett., 85, 1175 (2004).

[7] R.A. Minamisawa, M.J. Süess, R. Spolenak, J. Faist, C. David, J. Gobrecht, K.K. Bourdelle, H. Sigg. Nature Commun., 3, 1096 (2012).

[8] M.J. Süess, R. Geiger, R.A. Minamisawa, G. Schiefler, J. Frigerio, D. Chrastina, G. Isella, R. Spolenak, J. Faist, H. Sigg. Nature Photonics, 7, 466 (2013).

[9] D.S. Sukhdeo, D. Nam, J.-H. Kang, M.L. Brongersma, K.C. Saraswat. Photon. Res., 2, A8 (2014).

[10] A. Gassenq, K. Guilloy, G. Osvaldo Dias, N. Pauc, D. Rouchon, J.-M. Hartmann, J. Widiez, S. Tardif, F. Rieutord, J. Escalante, I. Duchemin, Y.-M. Niquet, R. Geiger, T. Zabel, H. Sigg, J. Faist, A. Chelnokov, V. Reboud, V. Calvo. Appl. Phys. Lett., 107, 191904 (2015).

[11] M. El Kurdi, M. Prost, A. Ghrib, S. Sauvage, X. Checoury, G. Beaudoin, I. Sagnes, G. Picardi, R. Ossikovski, P. Boucaud. ACS Photon., 3, 443 (2016).

[12] R.W. Millar, K. Gallacher, J. Frigerio, A. Ballabio, A. Bashir, I. Mac Laren, G. Isella, D.J. Paul. Opt. Express, 24, 4365 (2016).

[13] S. Bao, D. Kim, C. Onwukaeme, S. Gupta, K. Saraswat, K.H. Lee, Y. Kim, D. Min, Y. Jung, H. Qiu, H. Wang, E.A. Fitzgerald, C.S. Tan, D. Nam. Nature Commun., 8, 1845 (2017).

[14] A. Elbaz, M. El Kurdi, A. Aassime, S. Sauvage, X. Checoury, I. Sagnes, C. Baudot, F. Boeuf, P. Boucaud. APL Photon., 3, 106102 (2018).

[15] D. Nam, D.S. Sukhdeo, S. Gupta, J.-H. Kang, M.L. Brongersma, K.C. Saraswat. IEEE J. Select. Topics Quant. Electron., 20, 1500107 (2014).

[16] N. Tas, T. Sonnenberg, H. Jansen, R. Legtenberg, M. Elwenspoek. J. Micromech. Microeng., 6, 385 (1996).

[17] H.-C. Luan, D.R. Lim, K.K. Lee, K.M. Chen, J.G. Sandland, K. Wada, L.C. Kimerling. Appl. Phys. Lett., 75, 2909 (1999).

[18] J.-M. Hartmann, A. Abbadie, J.P. Barnes, J.M. Fedeli, T. Billon, L. Vivien. J. Cryst. Growth, 312, 532 (2010).

[19] Д.В. Юрасов, А.И. Бобров, В.М. Данильцев, А.В. Новиков, Д.А. Павлов, Е.В. Скороходов, М.В. Шалеев, П.А. Юнин. ФТП, 49, 1463 (2015).

[20] D.V. Yurasov, A.V. Antonov, M.N. Drozdov, V.B. Schmagin, K.E. Spirin, A.V. Novikov. J. Appl. Phys., 118, 145701 (2015).

[21] J. Liu, X. Sun, D. Pan, X. Wang, L.C. Kimerling, T.L. Koch, J. Michel. Opt. Express, 15, 11272 (2007).

[22] M.R. Barget, M. Virgilio, G. Capellini, Y. Yamamoto, T. Schroeder. J. Appl. Phys., 121, 245701 (2017).

[23] J. Frigerio, A. Ballabio, K. Gallacher, V. Giliberti, L. Baldassarre, R. Millar, R. Milazzo, L. Maiolo, A. Minotti, F. Bottegoni, P. Biagioni, D.J. Paul, M. Ortolani, A. Pecora, E. Napolitani, G. Isella. J. Phys. D: Appl. Phys., 50, 465103 (2017).

[24] Y. Yamamoto, M.R. Barget, G. Capellini, N. Taoka, M. Virgilio, P. Zaumseil, A. Hesse, T. Schroeder, B. Tillack. Mater. Sci. Semicond. Proc., 70, 111 (2017).

[25] D.V. Yurasov, A.V. Antonov, M.N. Drozdov, P.A. Yunin, B.A. Andreev, P.A. Bushuykin, N.A. Baydakova, A.V. Novikov. J. Cryst. Growth, 491, 26 (2018).

[26] D.V. Yurasov, A.V. Novikov, N.A. Baidakova, E.E. Morozova, P.A. Yunin, D.V. Shengurov, A.V. Antonov, M.N. Drozdov, Z.F. Krasilnik. Semicond. Sci. Technol., 33, 124019 (2018). 
[27] А.В. Новиков, Д.В. Юрасов, Е.Е. Морозова, Е.В. Скороходов, В.А. Вербус, А.Н. Яблонский, Н.А. Байдакова, Н.С. Гусев, К.Е. Кудрявцев, А.В. Нежданов, А.И. Машин. ФТП, 52, 1331 (2018).

[28] A. Gassenq, S. Tardif, K. Guilloy, I. Duchemin, N. Pauc, J.-M. Hartmann, D. Rouchon, J. Widiez, Y.M. Niquet, L. Milord, T. Zabel, H. Sigg, J. Faist, A. Chelnokov, F. Rieutord, V. Reboud, V. Calvo. J. Appl. Phys., 121, 055702 (2017).

[29] T.R. Hart, R.L. Aggarwal, B. Lax. Phys. Rev. B, 1, 638 (1970).

[30] S. Safran, B. Lax. J. Phys. Chem. Sol., 36, 753 (1975).

Редактор Л.В. Шаронова

\section{Locally strained Ge/SOI structures with improved heat sink as an active media for silicon optoelectronics}

D.V. Yurasov ${ }^{1}$, N.A. Baidakova ${ }^{1}$, V.A. Verbus ${ }^{1,2}$, N.S. Gusev ${ }^{1}$, A.I. Mashin ${ }^{3}$, E.E. Morozova ${ }^{1}$, A.V. Nezhdanov ${ }^{3}$, A.V. Novikov ${ }^{1,3}$, E.V. Skorohodov ${ }^{1}$, D.V. Shengurov ${ }^{1}$, A.N. Yablonskiy ${ }^{1}$

${ }^{1}$ Institute for Physics of Microstructures, Russian Academy of Sciences, 603950 Nizhny Novgorod, Russia

${ }^{2}$ National Research University Higher School of Economics, 603155 Nizhny Novgorod, Russia ${ }^{3}$ Lobachevsky State University of Nizhny Novgorod, 603950 Nizhny Novgorod, Russia

Abstract In this work formation of locally strained Ge structures on SOI (silicon on insulator) substrates is reported and their optical properties are discussed. Suspended Ge structures were fabricated by optical lithography, plasmachemical and wet chemical etching using the „stress concentration“ approach. The fabrication procedure of suspended structures was modified in such a way to provide the mechanical contact between them and the underlying layers so improving the heat dissipation from them. SOI substrates with top Si layer being only $100 \mathrm{~nm}$ thick were utilized in such fabrication scheme. The decrease of local heating in such kind of structures was confirmed by the study of micro-Raman scattering depending of scanning laser power. Micro-photoluminescence measurements have shown the remarkable enhancement of the integrated intensity from locally strained areas of a microstructure. It was also shown that structures brought in contact with underlying layers could sustain much higher pumping power densities without fracture as compared to the suspended ones. 